OPEN ACCESS

Edited by:

loan Opris,

University of Miami, United States

Reviewed by:

Mirjana Ratko Jovanovic,

Department of Psychiatry, Faculty of

Medical Sciences, University of

Kragujevac, Serbia

Xiaoli Li,

Beijing Normal University, China

*Correspondence:

Soyoung Yoo

mesoyoung@gmail.com

Specialty section: This article was submitted to

Neural Technology,

a section of the journal

Frontiers in Neuroscience

Received: 15 November 2017

Accepted: 15 February 2018

Published: 01 March 2018

Citation:

Jun J and Yoo S (2018) Three Research Strategies of Neuroscience and the Future of Legal Imaging

Evidence. Front. Neurosci. 12:120.

doi: 10.3389/fnins.2018.00120

\section{Three Research Strategies of Neuroscience and the Future of Legal Imaging Evidence}

\author{
Jinkwon Jun ${ }^{1}$ and Soyoung Yoo ${ }^{2,3 *}$ \\ ${ }^{1}$ KIAS Transdisciplinary Research Program, Korea Institute for Advanced Study, Seoul, South Korea, ${ }^{2}$ Human Research \\ Protection Center, Asan Medical Center, Seoul, South Korea, ${ }^{3}$ Health Innovation Big Data Center, Asan Medical Center, \\ Seoul, South Korea
}

Neuroscientific imaging evidence (NIE) has become an integral part of the criminal justice system in the United States. However, in most legal cases, NIE is submitted and used only to mitigate penalties because the court does not recognize it as substantial evidence, considering its lack of reliability. Nevertheless, we here discuss how neuroscience is expected to improve the use of NIE in the legal system. For this purpose, we classified the efforts of neuroscientists into three research strategies: cognitive subtraction, the data-driven approach, and the brain-manipulation approach. Cognitive subtraction is outdated and problematic; consequently, the court deemed it to be an inadequate approach in terms of legal evidence in 2012. In contrast, the data-driven and brain manipulation approaches, which are state-of-the-art approaches, have overcome the limitations of cognitive subtraction. The data-driven approach brings data science into the field and is benefiting immensely from the development of research platforms that allow automatized collection, analysis, and sharing of data. This broadens the scale of imaging evidence. The brain-manipulation approach uses high-functioning tools that facilitate non-invasive and precise human brain manipulation. These two approaches are expected to have synergistic effects. Neuroscience has strived to improve the evidential reliability of NIE, with considerable success. With the support of cutting-edge technologies, and the progress of these approaches, the evidential status of NIE will be improved and NIE will become an increasingly important part of legal practice.

Keywords: neuroscience and law, neurolaw, neuroethics, brain manipulation, brain function database

\section{INTRODUCTION: REQUIREMENTS FOR LEGAL IMAGING EVIDENCE}

Neuroscientific imaging evidence (NIE) has become an integral part of the criminal justice system in the United States. During 2005-2012, more than 1,500 judicial opinions have involved NIE (Presidential Commission for the Study of Bioethical Issues, 2015). However, in most legal cases, NIE is submitted and used only to mitigate penalties, because the court does not recognize it as substantial evidence (Salerno and Bottoms, 2009; Farahany, 2016). The incomplete acceptance of NIE is supported by criticisms regarding the validity of the imaging data (Aharoni et al., 2008; Pardo and Patterson, 2011; Jones et al., 2013; Buckholtz and Faigman, 2014; Dawid et al., 2014; Faigman et al., 2014), which have raised concerns regarding the evidential reliability of imaging evidence. 
For example, in People v. Ruiz, the accused claimed incompetence to stand trial on the basis of neuropsychological testimony ${ }^{1}$. Two experts examined Ruiz's brain and diagnosed him with a severe language disorder. Their opinion was based on neurobiological evidence supporting poor development of the left part of the brain, which is known to be associated with language skills. The trial judge concluded that the evidence supported the incompetence of the accused. This legal decision was based on the argument shown below.

Premise \#1: In the present case, the defendant has a deficit in brain area $\mathrm{B}$.

Premise \#2: In other studies, brain area $\mathrm{B}$ is putatively responsible for cognitive process $M$.

Conclusion: Thus, the damage in area $\mathrm{B}$ of the brain of the present case demonstrates his incompetence in cognitive process $\mathrm{M}$.

Premise \#1 is testable by investigation of the culprit; therefore, premise \#2 is the key for establishing the validity of the conclusion. To prove premise \#2, neuroscience should demonstrate that brain area $\mathrm{B}$ is indeed putatively responsible for cognitive process M. However, it is difficult to find a clear relationship between mental processes and brain regions. Critics have emphasized that there are numerous situations wherein premise \#2 can be discredited, such as degeneration (Friston et al., 2006) and modulation (Logothetis et al., 2001). Therefore, critics claim that most neuroimaging studies show only correlations, rather than causations (Aharoni et al., 2008; Buckholtz and Faigman, 2014). Moreover, they claim that neuroscience is incapable of confirming the role of a particular brain region in an individual act (Dawid et al., 2014; Faigman et al., 2014).

However, such arguments are misguided, because the law does not require complete proof of a causal relationship. The federal rule of evidence 401 defines the Test for Relevant Evidence as follows.

\section{Evidence is relevant if}

(a) it has any tendency to make a fact more or less probable than it would be without the evidence

Furthermore, the federal rule of evidence 702 defines the condition for Testimony by Expert Witness as follows:

(b) the testimony is based on sufficient facts or data;

(c) the testimony is the product of reliable principles and methods; and

(d) the expert has reliably applied the principles and methods to the facts of the case.

According to the federal rules of evidence, if sufficient data are gathered in accordance with reliable principles and methods, NIE can be considered significant evidence. The court is not concerned about the evidence being complete or incomplete. Accordingly, the degree of reliability of NIE is the actual issue.

From this perspective, the remarkable improvements in the field of neuroscience in recent years should be given

${ }^{1}$ People v. Ruiz. F057116. (Cal: Court of Appeal, 5th Dist., 2010). importance. Newly developed techniques and theoretical tools have contributed to an improvement in the evidential reliability of imaging data. Accordingly, the aim of this article is to explain how these developments are overcoming the reliability issues of NIE. We have classified the efforts of neuroscientists into three research strategies: cognitive subtraction, the data-driven approach, and the brain-manipulation approach. All strategies share the same goal of confirming whether brain area B putatively engages in cognitive process $M$ (hereafter referred to as $B \rightarrow M)^{2}$, which will consequently improve the reliability of premise \#2. The three approaches are presented in Table 1.

The first part of the article explains cognitive subtraction, which is the most common strategy in the field. However, it has limitations with regard to legal use, as pointed out by critics. Then, we introduce the data-driven and brain-manipulation approaches as more appropriate alternatives in the subsequent two sections. The data-driven approach brings data science into the field of imaging neuroscience. The brain-manipulation approach is based on novel methodologies for controlling the brain. In the second and third sections, we discuss how the datadriven and brain-manipulation approaches, respectively, can increase the reliability of NIE with the support of cutting-edge technologies. We conclude by asserting that criticisms against the legal use of NIE may no longer be appropriate because of advances in neuroscience.

\section{COGNITIVE SUBTRACTION AND ITS LIMITATIONS}

Most experimental designs in neuroscience research follow the following format. First, researchers use imaging technology, such as functional magnetic resonance imaging (fMRI) or positron emission tomography (PET), to scan brain activation patterns (such as the blood oxygen level-dependent [BOLD] signal) in subjects while they are not engaged in any kind of task. This is referred to as the resting state or baseline. The researchers then scan the brain while the subjects perform a cognitive task, such as face recognition, memory loading, or auditory stimulation. We can let $\mathrm{C} 0$ be the resting state condition and $\mathrm{C} 1$ be the condition where the brain is engaged in a psychological process of interest (mental process $\mathrm{M}$ ). The difference in the BOLD signal between $\mathrm{C} 0$ and $\mathrm{C} 1$ in brain region $\mathrm{B}$ is the measure of interest. This method for evaluating the relationship between brain and function is known as cognitive subtraction (Friston et al., 1996; Logothetis et al., 2001).

In Figure 1, the result of cognitive subtraction is interpreted as $\mathrm{R} 1$ engaging in a cognitive process related to $\mathrm{C} 1$, because the BOLD signal in $\mathrm{R} 1$ is significantly increased during $\mathrm{C} 1$ compared with that during $\mathrm{C} 0$. However, cognitive subtraction alone is not sufficient to confirm inference $B \rightarrow M$, because cognitive subtraction merely shows that brain area $\mathrm{B}$ is activated (inferentially, $\mathrm{M} \rightarrow \mathrm{B}$ ) during mental process $\mathrm{M}$. There are conditions that render $\mathrm{M} \rightarrow \mathrm{B}$ insufficient as evidence for $\mathrm{B} \rightarrow \mathrm{M}$.

\footnotetext{
${ }^{2} \mathrm{~B} \rightarrow \mathrm{M}$ is often referred as "reverse inference." This, however, is not only a broader concept than $\mathrm{B} \rightarrow \mathrm{M}$, but is also very technical; therefore, we intentionally avoid the use of this term. See Huettel et al. (2009).
} 
Figure 2A represents a case of degeneracy, wherein multiple brain regions engage in the same psychological process (Noppeney et al., 2004). The left parietal cortex and left putamen are examples of degeneracy, because both regions can be engaged in the cognitive process of reading, and the region activated during reading differs across individuals (Seghier et al., 2008). Therefore, it is difficult to determine the region responsible for reading using cognitive subtraction only. An example of correlation, which is illustrated by the dotted line in Figure 2B, could be neuromodulation, which is an activation that facilitates control of other brain regions. In Figure 2B, while activation of $\mathrm{B} 2$ is the actual cause of $\mathrm{M}$, activation of $\mathrm{B} 1$ is a mere response to the change in B2. According to Logothetis (2008), the majority of neurons do not deliver a signal; instead, they modulate the activity of other neurons. This may lead to an incorrect interpretation that $\mathrm{B} 1$ is the area responsive to experimental stimuli. However, when $\mathrm{B} 1$ is damaged, process $\mathrm{M}$ remains intact.

In both cases, cognitive subtraction can fallaciously judge that $\mathrm{B} 1$ is engaged in process $\mathrm{M}$. However, neuroscientists are aware of this problem and have also attempted to resolve it. The conventional manner of dealing with this issue is known as dissociation (Teuber, 1955; Shallice, 1988). Dissociation methods aim to ascertain whether two brain regions are underwritten by the same process (Henson, 2005, 2006a,b). The basic logic of dissociation is to exclude fallacious possibilities, such as those described for the cases in Figure 2, using additional experimental

TABLE 1 | Research strategies for $B \rightarrow M$.

\begin{tabular}{ll}
\hline Strategy & Method \\
\hline Cognitive Subtraction & $\begin{array}{l}\text { Manipulation of cognitive process M and } \\
\text { observation of brain area B }\end{array}$ \\
Data-Driven Approach & $\begin{array}{l}\text { Statistical processing of data obtained from } \\
\text { cognitive subtraction }\end{array}$ \\
Brain-Manipulation Approach & $\begin{array}{l}\text { Manipulation of brain area B and observation of } \\
\text { cognitive process M }\end{array}$
\end{tabular}

data. For example, assume that $\mathrm{C} 0$ is the condition-related mental function F0 and $\mathrm{C} 1$ is the condition-related function F1. When the activation pattern of R1 and R2 on F1 and F2 is as shown in Figure 3A, the relationship between R1 and R2 is called double dissociation (Henson, 2005). If R1 is only engaged in F0 and R2 is only engaged in $\mathrm{F} 1$, there is evidence indicating that the two regions are not engaged in the same job. Based on this logic, researchers can separate the brain area with the function from the irrelevant areas. However, double dissociation is not sufficient to draw the conclusion that $\mathrm{B} \rightarrow \mathrm{M}$ because it cannot exclude the possibility that R1 and R2 are antagonistic. To resolve this, at least three experimental conditions are required. Let $\mathrm{C} 0$ be the control condition, $\mathrm{C} 1$ be the condition that is less engaged in cognitive process $\mathrm{M}$, and $\mathrm{C} 2$ be the condition that is more engaged in function $\mathrm{F}$ (i.e., assuming a linearity among $\mathrm{C} 0, \mathrm{C} 1$, and $\mathrm{C} 2$ ). Then, if the activation patterns are as shown in Figure 3C, which are collectively called reverse association (Dunn and Kirsner, 1988; Henson, 2005; Machery, 2012, 2014), R1 and R2 are not antagonistic. If they suppress each other, the result would be as shown in Figure 3B, not as shown in Figure 3C.

In this manner, neuroscientists can improve the reliability of imaging data by accumulating experimental data from various conditions. This research strategy, often referred to as imaging dissociation (Machery, 2012), is the basic strategy used to
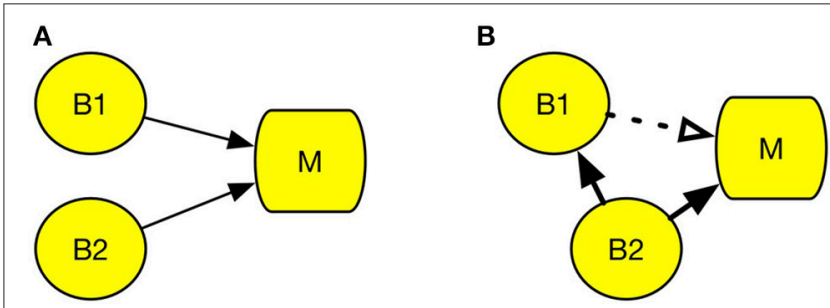

FIGURE 2 | Conditions that undermine cognitive subtraction. (A) Degeneracy. (B) Correlation. Circle $\mathrm{B}$ refers to a specific brain region and square $\mathrm{M}$ refers to a specific psychological process. The solid line arrow indicates a causal relation and the dotted line arrow indicates a correlation.

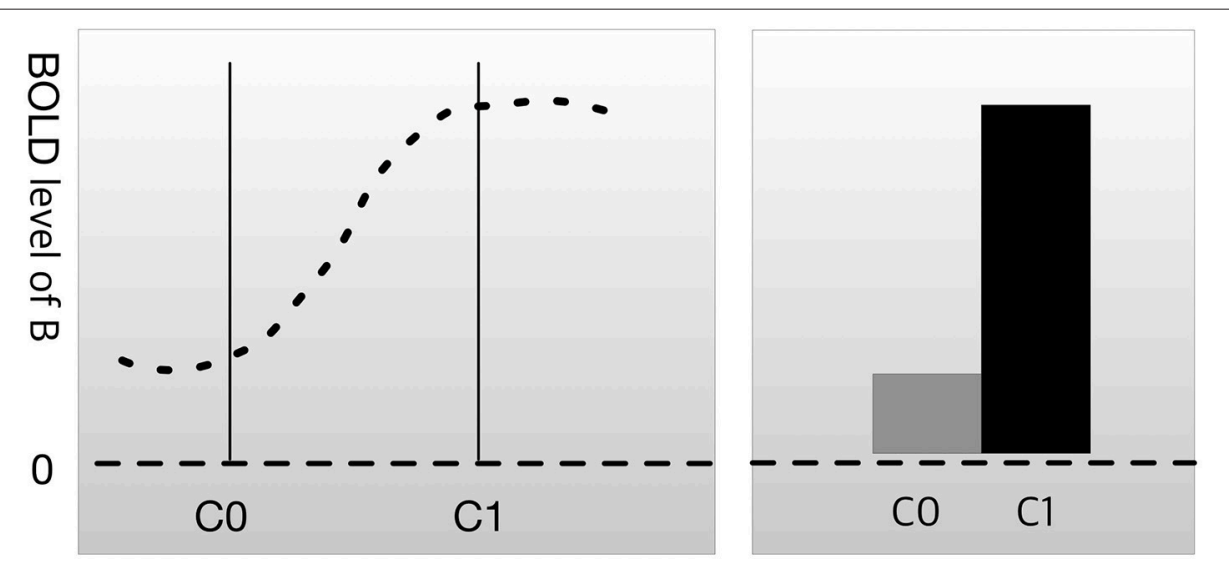

FIGURE 1 | Cognitive subtraction. $\mathrm{C} 0$ is a control condition, and $\mathrm{C} 1$ is an experimental condition. The height of the graph represents the level of the blood oxygen level-dependent (BOLD) signal in brain region $\mathrm{B}$. The difference in the BOLD signal between C0 and C1 is considered to indicate that brain region B is engaged in C1. 


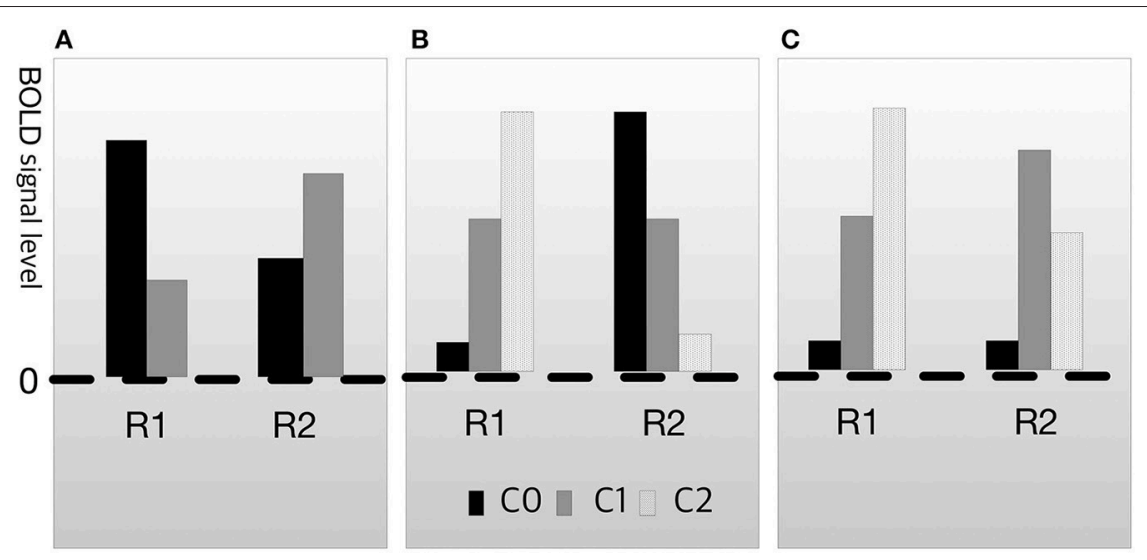

FIGURE 3 | Types of dissociation. (A) Double dissociation. (B) Crossover double association. (C) Reverse associateon.

elucidate the relationship between the brain area and cognitive function. However, it should be noted that this approach also cannot provide a complete confirmation of $\mathrm{B} \rightarrow \mathrm{M}$ (Friston et al., 2006; Poldrack, 2006; Logothetis, 2008; Anderson, 2010; Sternberg, 2011). The ultimate reason is that the human brain is complex; therefore, there are near-infinite conditions for confirming $\mathrm{B} \rightarrow \mathrm{M}$, and testing of all conditions is extremely demanding (Aguirre et al., 2003).

Nevertheless, scientific evidence and other evidence do not have to be perfectly confirmed in order to have legal reliability. For example, DNA fingerprinting is now widely accepted as substantial evidence, even though it is not incontrovertible. When DNA evidence is properly collected and analyzed by qualified experts, its credibility is admitted by the court (National Research Council, 2011). The same principle should be applied to neuroscientific evidence.

The Daubert standard, which has become the benchmark test for the reliability of neuroscientific evidence, has set the conditions for the legal use of NIE as follows:

(1) whether the theory or technique can be tested and has been tested

(2) whether the theory or technique has been subjected to peer review and publication

(3) the known or potential rate of error of the method used and the existence and maintenance of standards controlling the technique's operation

(4) whether the theory or method has been generally accepted by the scientific community ${ }^{3}$.

In 2012, in United States v. Semrau, the Supreme Court decided that NIE for lie detection, which is mainly based on cognitive subtraction, satisfies conditions (1) and (2), but not conditions (3) and (4) ${ }^{4}$.

As the sentence shows, NIE has not reached the level achieved by DNA evidence at present. However, the decision left a caveat for the future.

${ }^{3}$ Daubert v. Merrell Dow Pharmaceuticals 509 U.S. 579 (1993). p. 593-594.

${ }^{4}$ United States v. Semrau, 693 F.3d 510 (6th Cir. 2012), p. 31.
In the future, should fMRI-based lie detection undergo further testing, development, and peer review, improve upon standards controlling the technique's operation, and gain acceptance by the scientific community for use in the real world, this methodology may be found to be admissible even if the error rate is not able to be quantified in a real world setting ${ }^{4}$.

As stated above, if neuroscience research succeeds in improving the reliability of NIE, it will be accepted as substantial evidence. It is thus important to understand the problems involved in cognitive subtraction. The first limitation is related to the issue of causality. In a normal experimental setting, brain area $B$ is the cause and cognitive process $M$ is the effect. This indicates that cognitive subtraction is a method for manipulating the effect and observing the cause. Generally, discerning the cause from the effect is difficult, particularly in cases involving a complex structure. Second, establishment of a reliable conclusion regarding $\mathrm{B} \rightarrow \mathrm{M}$ via cognitive subtraction is a very demanding process, because the human brain is complex and much information is required to prove that $\mathrm{B} \rightarrow \mathrm{M}$.

Fortunately, the two major drawbacks of cognitive subtraction are being resolved because of advances in the field of neuroscience, which can be characterized by two trends: the datadriven approach and the brain-manipulation approach. These two strategies illustrate how neuroscientists have attempted to deal with the reliability issue pertaining to imaging evidence for $\mathrm{B} \rightarrow \mathrm{M}$. In the next two sections, we introduce and describe stateof-the-art scientific progress in these areas and discuss how it can shed light on the future of NIE.

\section{DATA-DRIVEN APPROACH}

As discussed above, cognitive subtraction is not a suitable strategy for obtaining legal imaging evidence. One problem is that the collection of brain-function data via dissociation is a very demanding and labor-intensive process. However, the data-driven approach tackles this issue from a different angle with the help of data science. The core concept of the datadriven approach is that a sufficiently large amount of information can substantially increase the reliability of the brain-function 
relationship. Accordingly, this approach utilizes large databases and computerized data processing (Yarkoni et al., 2011; Hutzler, 2014; Poldrack and Yarkoni, 2016), thus justifying its name.

The basic logic of the data-driven approach can be summarized as follows.

If a region is active across more psychological functions, it will provide less support for $\mathrm{B} \rightarrow \mathrm{M}$.

If a region is active across fewer psychological functions, it will provide more support for $\mathrm{B} \rightarrow \mathrm{M}$.

The idea is simple. If a region is engaged in only one mental process, its activation would provide good evidence for the occurrence of this mental process. However, if the region is engaged in various mental processes, then its activation would not yield good evidence for the occurrence of the mental process. Among the various implements for this idea, we will focus on the Bayesian approach ${ }^{5}$ which is based on the use of the Bayesian rule to represent and calculate the reliability of evidential support:

$$
P(M \mid B)=\frac{P(B \mid M) P(M)}{P(B \mid M) P(M)+P(B \mid \sim M) P(\sim M)}_{6}^{6}
$$

Bayesian rule for $\mathrm{B} \rightarrow \mathrm{M}$

Equation 1 is the Bayesian rule for measuring the certainty of regional data obtained using imaging technology (Poldrack, 2006; Yarkoni et al., 2011; Rubin et al., 2016). M refers to the ongoing mental process and $\mathrm{B}$ refers to activation of the region of interest. $P(M \mid B)$ is the conditional probability of occurrence of psychological process $M$ on activation of brain region $B$, which is interpreted as the degree of credibility of the inference $\mathrm{B} \rightarrow \mathrm{M} . P(M)$ is the base rate for mental process $\mathrm{M}, P(B \mid M)$ is the conditional probability of activation of brain region $B$ on occurrence of psychological process $\mathrm{M}$, and $P(B \mid \sim M)$ is the conditional probability of activation of region $\mathrm{B}$ without the occurrence of psychological process $M$. The usefulness of the Bayesian rule is that it provides an update on the present degree of reliability from new evidence. When a task relevant to $M$ induces activation of $\mathrm{A}, P(B \mid M)$ should increase. Conversely, when a task that is irrelevant to $\mathrm{M}$ induces activation of $\mathrm{A}, P(B \mid \sim ! M)$ should increase. If $P(B \mid M)$ increases, then $P(M \mid B)$ also increases; in contrast, if $P(B \mid \sim ! M)$ increases, then $P(M \mid B)$ decreases. In particular, it is important that $P(B \mid M)$ and $P(B \mid \sim ! M)$ data can be obtained via cognitive subtraction.

In this way, the Bayesian approach can mediate data obtained from cognitive subtraction to $\mathrm{B} \rightarrow \mathrm{M}$. However, even though this approach works well, it is impractical to use a single study to compare the various conditions for adequate justification of $\mathrm{B} \rightarrow \mathrm{M}$. Meta-analyses, which depend on manpower, cannot avoid the scalability problem, because humans can only process a limited amount of data. However, the data-driven approach, which utilizes data science technology, such as machine learning and artificial intelligence, is not limited by the information processing capacity. Recently, studies in neuroscience established brain-function databases, such as BrainMap (Laird et al.,

${ }^{5}$ The likelihood approach may be another influential method. See Machery (2014). ${ }^{6}$ This equation is adopted from Poldrack (2006).
2005), Brede (Nielsen et al., 2004), SuMS (Dickson et al., 2001), OpenfMRI (Poldrack, 2011), NeuroVault (Gorgolewski et al., 2015). All these are automated platforms for data collection, storage, and analysis. For example, Neurosynth (http://www.neurosynth.org) uses data mining technology and a computational linguistic method for the automatic extraction of information from published articles and reports, ultimately generating clear, understandable images from the gathered information (Yarkoni et al., 2011). Currently, the Neurosynth database contains more than 10,000 articles and 36,000 discrete activation patterns. Furthermore, brain imaging standards such as the Neuroimaging Data Model (http://www.nidm.nidash.org) and the Nipype (Gorgolewski et al., 2011) are being developed; these will provide fully automatic, reproducible, shareable, and open-source analysis pipelines. With large databases such as these, neuroscientists are now trying to expand research in imaging science from region-level studies to whole brain-level studies (Del Pinal and Nathan, 2017). This approach is aiding in determination of the relationships among multiple brain regions and cognitive function in order to overcome the issue of the lack of specificity (Nathan and Del Pinal, 2017).

With regard to the legal context for NIE, it is important that these databases and analysis programs provide a criterion for evaluating the strength of $\mathrm{B} \rightarrow \mathrm{M}$. Bayesian statistics conventionally use the Bayes factor for evaluating the conclusion. Table 2 shows an example of the assessment criteria (Kass and Raftery, 1995; Jeffreys, 1998).

In case of Equation (1), the Bayes factor is $\frac{P(A \mid M)}{P(A \mid \sim M)}$, and the value of this formula is understood as the reliability of the imaging evidence. For example, Poldrack (2006) tested whether activation of Broca's area is engaged in language function using the BrainMap database. They derived a Bayes factor of 2.3, which was below 3.2 and consequently considered to represent weak evidence. In contrast, the ventral striatum data from the study by Ariely and Berns (2010) had a Bayes factor of 9, which was considered to represent moderately strong evidence.

In this manner, the data-driven approach can provide an error estimation for $\mathrm{B} \rightarrow \mathrm{M}$. It will grade the evidence for the degeneration case in Figure 2A as untrustworthy, because $\mathrm{M}$ was associated with B1 in some cases and B2 in some. It provides an important advantage for NIE, because the Daubert standard requires estimation of the error rate.

The known or potential rate of error of the method used and the existence and maintenance of standards controlling the technique's operation $^{3}$.

The data-driven approach can compensate for this limitation of NIE. Accordingly, a large brain-function database will lead to progress in the legal use of NIE. Moreover, the technology for

TABLE 2 | Assessment table for the Bayes factor.

\begin{tabular}{ll}
\hline Value of the Bayes factor & Reliability of the inference \\
\hline $1-3.2$ & Not worth more than a bare mention \\
$3.2-10$ & Substantial \\
$10-100$ & Strong \\
$>100$ & Decisive
\end{tabular}


data storage and analysis accelerates progress, thereby leveraging the scale and breadth of imaging data and increasing the power of the data-driven approach with the passage of time.

\section{BRAIN-MANIPULATION APPROACH}

The issue of causality with NIE is commonly pointed out by critics (Feigenson, 2007; Aharoni et al., 2008). However, the brain-manipulation approach is a crucial advancement for resolution of this problem. Conventionally, the basic idea of causality in science is paralleled by intervention of the antecedents (Woodward, 2005). For imaging science, the antecedent is the brain area. As discussed before, cognitive subtraction merely controls the consequence (i.e., the cognitive process), which is the reason for it being insufficient to confirm causality.

If the activation of a specific brain region can be freely manipulated, direct testing of $\mathrm{B} \rightarrow \mathrm{M}$ becomes possible. However, conventional brain-manipulation methodologies have at least two major obstacles. First, they are mostly invasive, indicating that they can result in irreversible damage to the subject. Because there is no scientific consensus that animals can perform moral or ethical cognitive processes, legal imaging evidence should be collected from human subjects. Although various brain manipulation techniques are available, most of them require cranial surgery or may possibly damage the brain tissues. Therefore, such methods cannot be applied to humans for ethical reasons. Second, conventional methods are associated with a low spatiotemporal resolution. NIE for legal issues have highly sophisticated functions such as moral decision making, social relationships, and impulse control, among others. Because these cognitive processes are implemented by complex neuronal networks, accurate manipulation methods are necessary. For example, in lesional studies employing a conventional manipulation method, a patient with brain damage is compared to a healthy individual. Generally, a lesion includes a large number of neurons; therefore, mental processes cannot be accurately distinguished.

However, the field of neuroscience has developed safe and precise methods for brain manipulation. The brain-manipulation approach depends on state-of-the-art technologies, such as electric current stimulation, transcranial magnetic stimulation (TMS), optogenetics, and ultrasound, among others. Electric current stimulation, which uses a weak electric current for activation or deactivation of neurons, has several variations. Implanted microelectrode arrays or deep brain stimulation (DBS) utilize a chip of electrodes to stimulate a certain brain region. DBS is not only recognized as a safe instrument, but is also widely used for the treatment of Alzheimer's disease or treatment-resistant depression (Benabid et al., 2009). It can also stimulate subcortical regions. However, implantation methods still require head surgery. In contrast, transcranial direct current stimulation (tDCS) and its variations (transcranial alternating current stimulation, transcranial random noise stimulation, etc.) do not require cranial surgery. For typical tDCS, a large pad $(\sim 25 \mathrm{~cm})$ is attached to the skin on the head for delivery of an electrical current. High-definition tDCS, which was recently developed (Nitsche et al., 2008; Caparelli-Daquer et al., 2012), provides much better spatial resolution of $\sim 1 \mathrm{~cm}^{2}$. TMS is expensive and requires a trained technician; however, it provides better spatiotemporal resolution than tDCS. Instead of direct delivery of the current, TMS uses electromagnetic induction. The coil, which is placed near the head of the subject, generates an electromagnetic field and produces a weak current in the target region of the brain. High-definition TMS provides a spatial resolution of $\sim 0.5-1 \mathrm{~cm}^{2}$ (Sliwinska et al., 2014), whereas newly developed micromagnetic stimulation coils provide a spatial resolution of $\sim 500 \mu \mathrm{m}$ (Bonmassar et al., 2012).

Other cutting-edge techniques are also overcoming the limitations of the conventional methods (Lewis et al., 2016). Optogenetics uses genetically modified neurons that express light-sensitive channel proteins on their membrane; therefore, researchers can control their activation or deactivation using light. It provides a surprisingly high spatiotemporal resolution (Aston-Jones and Deisseroth, 2013; Häusser, 2014; Adamantidis et al., 2015; Deisseroth, 2015). Recently developed organic lightemitting diode arrays have $6 \times 9-\mu \mathrm{m}^{2}$ elements, which are smaller than a neuron (Steude et al., 2016). However, there is an important hurdle for application of this method to humans, because optogenetics requires genetic modification. However, viral vectors are known to be relatively safe for consideration in clinical trials (Gilbert et al., 2014). Noninvasive delivery methods are also under development (Wang et al., 2017). Ultrasound methods thus have the advantage of being noninvasive. They use a mechanical pressure wave (sound wave) with a high frequency $(>20 \mathrm{kHz}$ ), which can be transmitted through solid structures, including bone and soft tissues. Intensive ultrasound (over 1 $\mathrm{w} / \mathrm{cm}^{2}$ ) controls neuronal excitation by producing thermal effects (Tufail et al., 2010, 2011). Studies on ultrasound techniques have reported spatial resolutions $<3 \mathrm{~mm}^{2}$. Researchers are aiming to select brain regions $<1 \mathrm{~mm}^{2}$, which is five times better resolution than that achieved by TMS. Another method attracting attention is temporal interference (TI) stimulation (Grossman et al., 2017). In this approach, researchers use two pairs of surface electrodes to generate 2 and $2.01-\mathrm{kHz}$ sinusoidal stimulations concurrently, and the envelope of the two electrical stimulations results in a 10$\mathrm{Hz}$ beat frequency in the deep brain region. While a neuron does not respond to a high-frequency stimulation, it does respond to a low-frequency stimulation. Using this feature, TI manipulates the deep brain region in a noninvasive manner. Neuroscientists are now developing a TI stimulation approach that uses multiple electrodes in order to expand its resolution. Many have deemed this technology as a breakthrough in the field of neuronal modulation (Dmochowski and Bikson, 2017).

We summarized the brain manipulation technologies in Table 3. The future of the brain-manipulation approach looks promising. It cannot be said that these methods have completely conquered the long-standing obstacles for brain control, but they are rapidly improving and neuroscientists and engineers are continuing to invent new technology. Neuroscience has already applied manipulation methods with imaging techniques (Wagner et al., 2007; Bestmann and Feredoes, 2013), for example, TMS with fMRI (Bestmann et al., 2008; Ruff et al., 2009; Siebner et al., 2009) and optogenetics with fMRI (Lin et al., 2016). With regard 
TABLE 3 | Brain manipulation methods.

\begin{tabular}{lll}
\hline Method & Non-invasiveness & Spatial resolution \\
\hline Legion study & Yes & Very low \\
Deep brain stimulation & No & Low (can stimulate \\
& & deep brain areas) \\
Transcranial direct current stimulation & Yes & $<1 \mathrm{~cm}^{2}$ \\
Transcranial Magnetic Stimulation & Yes & $0.5-1 \mathrm{~cm}^{2}$ \\
Optogenetics & No & Very high \\
Ultrasound & Yes & $1-3 \mathrm{~mm}^{2}$ \\
TI (temporal interference) stimulation & Yes & Low (can stimulate \\
& & deep brain areas) \\
\hline
\end{tabular}

to methodological improvement, the manipulation approach is expected to be useful for solving the issue of causality (Dijkstra and de Bruin, 2016). For example, manipulation tests can rule out the correlational error in Figure 2B. If we manipulate B1, then no change will be observed in $\mathrm{M}$; thus, we can conclude that $\mathrm{B} 1 \rightarrow \mathrm{M}$ is a mere correlation. Henson mentioned that "imaging data from an experimental manipulation ... are no more correlational" (Henson, 2005, p. 222), indicating that the results obtained from manipulation will be much more reliable than correlations.

In addition, manipulation methods can complement the datadriven approach. For example, dynamic causal models (DCMs) are used to test the interaction of TMS-induced neuronal changes with cognition (Esser et al., 2005; Cona et al., 2011). A neural model built by DCMs predicts the impact of physiological signals on cognition, and researchers can manipulate the brain region to test its prediction. This illustrates how manipulation and neuroimaging can be used together. Confirmation of results obtained using the data-driven approach with the manipulation approach will further increase the reliability of NIE. Thus, these two research strategies can exhibit synergistic effects.

\section{CONCLUSION: DEVELOPMENT OF NEUROSCIENCE AND FUTURE OF LEGAL NIE}

We described three neuroimaging strategies for legal use. The first strategy, cognitive subtraction, is a relatively old and problematic approach and is considered unreliable. The other two approaches, the data-driven and brain-manipulation approaches, are rapidly overcoming the limitations of cognitive subtraction. Critics assert that there is a significant gap between NIE and legal evidence, and that neuroscience can only

\section{REFERENCES}

Adamantidis, A., Arber, S., Bains, J. S., Bamberg, E., Bonci, A., Buzsaki, G., et al. (2015). Optogenetics: 10 years after ChR2 in neurons-views from the community. Nat. Neurosci. 18, 1202-1212. doi: 10.1038/ nn. 4106

Aguirre, G. K., Feinberg, F. T. E., and Farah, M. J. (2003). "Functional imaging in behavioral neurology and cognitive neuropsychology," in Behavioral Neurology and Neuropsychology, eds T. E. Feinberg and M. J. Farah (New York, NY: McGraw-Hill), 35-46. demonstrate correlations (Aharoni et al., 2008). Furthermore, these two types of evidence have different purposes (Dawid et al., 2014) and are consequently incommensurable (Buckholtz and Faigman, 2014). However, in the present article, we have shown that neuroscience and the law of evidence pursue the same goal, which is to confirm the evidential reliability of NIE. Cutting-edge technologies increase the possibility of achieving this common goal. We therefore argue that the gap pointed out by critics is being narrowed by the efforts of neuroscientists.

We are convinced that both approaches will be applied in legal practice in the near future. For example, Gur and his colleagues at the University of Pennsylvania established a standard procedure for the legal use of NIE, which is called the Neuroforensics Service (Gur et al., 2016). They constructed the computerized neurocognitive battery (CNB) for analyzing the brain-function relationship (Gur et al., 2010). We want to emphasize that CNB can be significantly improved by application of both aforementioned approaches. First, because it is not an automated database, it may significantly benefit from the datadriven approach. A large brain-function database built with data technology can provide a wider range of data and better error estimates. Second, the manipulation method can provide an effective re-examination of known data, thereby making CNB more trustworthy. Furthermore, in cases where no data are available, a manipulation experiment can provide an appropriate solution.

Therefore, the two trends in neuroscience development are likely to make the legal use of NIE more reliable. Neuroscientists have striven to improve the evidential reliability of NIE and have achieved considerable success. As their research progresses, NIE will become an increasingly important part of legal practice.

\section{AUTHOR CONTRIBUTIONS}

JJ provided the core idea and wrote the draft of the article. SY developed the idea, provided the summary of technological details, and approved the final version of the manuscript.

\section{ACKNOWLEDGMENTS}

The authors thank Hyemin Han (The University of Alabama) and Minryung Song (KAIST) for their valuable advice. They would particularly like to thank Edouard Machery (University of Pittsburgh), who taught them the logic of imaging technology. Finally, the authors thank Dr. Joon Seo Lim from the Scientific Publications Team at Asan Medical Center for his editorial assistance in preparing this manuscript.

Aharoni, E., Funk, C., Sinnott-Armstrong, W., and Gazzaniga, M. (2008). Can neurological evidence help courts assess criminal responsibility? Lessons from law and neuroscience. Ann. N.Y. Acad. Sci. 1124, 145-160. doi: 10.1196/annals.1440.007

Anderson, M. L. (2010). Neural reuse: a fundamental organizational principle of the brain. Behav. Brain Sci. 33, 245-266; discussion 266-313. doi: 10.1017/S0140525X10000853

Ariely, D., and Berns, G. S. (2010). Neuromarketing: the hope and hype of neuroimaging in business. Nat. Rev. Neurosci. 11, 284-292. doi: $10.1038 / \mathrm{nrn} 2795$ 
Aston-Jones, G., and Deisseroth, K. (2013). Recent advances in optogenetics and pharmacogenetics. Brain Res. 1511, 1-5. doi: 10.1016/j.brainres.2013.01.026

Benabid, A. L., Chabardes, S., Mitrofanis, J., and Pollak, P. (2009). Deep brain stimulation of the subthalamic nucleus for the treatment of Parkinson's disease. Lancet Neurol. 8, 67-81. doi: 10.1016/S1474-4422(08)70291-6

Bestmann, S., and Feredoes, E. (2013). Combined neurostimulation and neuroimaging in cognitive neuroscience: past, present, and future. Ann. N.Y. Acad. Sci. 1296, 11-30. doi: 10.1111/nyas.12110

Bestmann, S., Ruff, C. C., Blankenburg, F., Weiskopf, N., Driver, J., and Rothwell, J. C. (2008). Mapping causal interregional influences with concurrent TMS-fMRI. Exp. Brain Res. 191, 383-402. doi: 10.1007/s00221-008-1601-8

Bonmassar, G., Lee, S. W., Freeman, D. K., Polasek, M., Fried, S. I., and Gale, J. T. (2012). Microscopic magnetic stimulation of neural tissue. Nat. Commun. 3:921. doi: $10.1038 /$ ncomms 1914

Buckholtz, J. W., and Faigman, D. L. (2014). Promises, promises for neuroscience and law. Curr. Biol. 24, R861-R867. doi: 10.1016/j.cub.2014.07.057

Caparelli-Daquer, E. M., Zimmermann, T. J., Mooshagian, E., Parra, L. C., Rice, J. K., Datta, A., et al. (2012). A pilot study on effects of $4 \mathrm{x} 1$ high-definition tDCS on motor cortex excitability. Conf. Proc. IEEE Eng. Med. Biol. Soc. 2012, 735-738. doi: 10.1109/EMBC.2012.6346036

Cona, F., Zavaglia, M., Massimini, M., Rosanova, M., and Ursino, M. (2011). A neural mass model of interconnected regions simulates rhythm propagation observed via TMS-EEG. Neuroimage 57, 1045-1058. doi: 10.1016/j.neuroimage.2011.05.007

Dawid, A. P., Faigman, D. L., and Fienberg, S. E. (2014). Fitting science into legal contexts. Sociol. Methods Res. 43, 359-390. doi: 10.1177/0049124113515188

Deisseroth, K. (2015). Optogenetics: 10 years of microbial opsins in neuroscience. Nat. Neurosci. 18, 1213-1225. doi: 10.1038/nn.4091

Del Pinal, G., and Nathan, M. J. (2017). "Two kinds of reverse inference in cognitive neuroscience," in The Human Sciences after the Decade of the Brain, eds J. Leefmann and E. Hildt (London: Academic Press), 121-139.

Dickson, J., Drury, H., and Van Essen, D. C. (2001). 'The surface management system' (SuMS) database: a surface-based database to aid cortical surface reconstruction, visualization and analysis. Philos. Trans. R. Soc. Lond. B. Biol. Sci. 356, 1277-1292. doi: 10.1098/rstb.2001.0913

Dijkstra, N., and de Bruin, L. (2016). Cognitive neuroscience and causal inference: implications for psychiatry. Front. Psychiatry 7:129. doi: 10.3389/fpsyt.2016.00129

Dmochowski, J., and Bikson, M. (2017). Noninvasive neuromodulation goes deep. Cell 169, 977-978. doi: 10.1016/j.cell.2017.05.017

Dunn, J. C., and Kirsner, K. (1988). Discovering functionally independent mental processes: the principle of reversed association. Psychol. Rev. 95, 91-101. doi: 10.1037/0033-295X.95.1.91

Esser, S. K., Hill, S. L., and Tononi, G. (2005). Modeling the effects of transcranial magnetic stimulation on cortical circuits. J. Neurophysiol. 94, 622-639. doi: $10.1152 /$ jn. 01230.2004

Faigman, D. L., Monahan, J., and Slobogin, C. (2014). Group to Individual (G2i) Inference in Scientific Expert Testimony. Univ. Chicago Law Rev. 81, 417-480. doi: $10.2139 /$ ssrn.2298909

Farahany, N. A. (2016). Neuroscience and behavioral genetics in US criminal law: an empirical analysis. J. Law. Biosci. 2, 485-509. doi: 10.1093/jlb/lsv059

Feigenson, N. (2007). Brain imaging and courtroom evidence: on the admissibility and persuasiveness of fMRI. Int. J. Law Context 2, 233-255. doi: $10.1017 / S 174455230600303 \mathrm{X}$

Friston, K. J., Price, C. J., Fletcher, P., Moore, C., Frackowiak, R. S., and Dolan, R. J. (1996). The trouble with cognitive subtraction. Neuroimage 4, 97-104. doi: 10.1006/nimg.1996.0033

Friston, K. J., Rotshtein, P., Geng, J. J., Sterzer, P., and Henson, R. N. (2006). A critique of functional localisers. Neuroimage 30, 1077-1087. doi: 10.1016/j.neuroimage.2005.08.012

Gilbert, F., Harris, A. R., and Kapsa, R. M. I. (2014). Controlling brain cells with light: ethical considerations for optogenetic clinical trials. AJOB Neurosci. 5, 3-11. doi: 10.1080/21507740.2014.911213

Gorgolewski, K., Burns, C. D., Madison, C., Clark, D., Halchenko, Y. O., Waskom, M. L., et al. (2011). Nipype: a flexible, lightweight and extensible neuroimaging data processing framework in python. Front. Neuroinform. 5:13. doi: $10.3389 /$ fninf.2011.00013
Gorgolewski, K. J., Varoquaux, G., Rivera, G., Schwarz, Y., Ghosh, S. S., Maumet, C., et al. (2015). NeuroVault.org: a web-based repository for collecting and sharing unthresholded statistical maps of the human brain. Front. Neuroinform. 9:8. doi: 10.3389/fninf.2015.00008

Grossman, N., Bono, D., Dedic, N., Kodandaramaiah, S. B., Rudenko, A., Suk, H. J., et al. (2017). Noninvasive deep brain stimulation via temporally interfering electric fields. Cell 169, 1029.e1016-1041.e1016. doi: 10.1016/j.cell.2017. 05.024

Gur, R. C., Gur, O. M., Gur, A. E., and Gur, A. G. (2016). A Perspective on the Potential Role of Neuroscience in the Court, 85 Fordham, L. Rev. 547. Available online at: http://ir.lawnet.fordham.edu/flr/vol85/iss2/8 (Accessed October 16, 2017).

Gur, R. C., Richard, J., Hughett, P., Calkins, M. E., Macy, L., Bilker, W. B., et al. (2010). A cognitive neuroscience-based computerized battery for efficient measurement of individual differences: standardization and initial construct validation. J. Neurosci. Methods 187, 254-262. doi: 10.1016/j.jneumeth.2009.11.017

Häusser, M. (2014). Optogenetics: the age of light. Nat. Methods 11, 1012-1014. doi: 10.1038/nmeth.3111

Henson, R. (2005). What can functional neuroimaging tell the experimental psychologist? Q. J. Exp. Psychol. A 58, 193-233. doi: $10.1080 / 02724980443000502$

Henson, R. (2006a). Forward inference using functional neuroimaging: dissociations versus associations. Trends Cogn. Sci. 10, 64-69. doi: 10.1016/j.tics.2005.12.005

Henson, R. (2006b). What has (neuro)psychology told us about the mind (so far)? A reply to Coltheart (2006). Cortex 42, 387-392. doi: 10.1016/S0010-9452(08)70365-4

Huettel, S. A., Allen, W. S., and Gregory, M. (2009). Functional Magnetic Resonance Imaging. Sunderland: Sinauer.

Hutzler, F. (2014). Reverse inference is not a fallacy per se: cognitive processes can be inferred from functional imaging data. Neuroimage 84, 1061-1069. doi: 10.1016/j.neuroimage.2012.12.075

Jeffreys, H. (1998). The Theory of Probability. New York, NY: OUP Oxford.

Jones, O. D., Wagner, A. D., Faigman, D. L., and Raichle, M. E. (2013). Neuroscientists in court. Nat. Rev. Neurosci. 14, 730-736. doi: 10.1038/ nrn3585

Kass, R. E., and Raftery, A. E. (1995). Bayes factors. J. Am. Stat. Assoc. 90, 773-795. doi: 10.1080/01621459.1995.10476572

Laird, A. R., Lancaster, J. L., and Fox, P. T. (2005). BrainMap: the social evolution of a human brain mapping database. Neuroinformatics 3, 65-78. doi: $10.1385 / \mathrm{NI}: 3: 1: 065$

Lewis, P. M., Thomson, R. H., Rosenfeld, J. V., and Fitzgerald, P. B. (2016). Brain neuromodulation techniques: a review. Neuroscientist 22, 406-421. doi: $10.1177 / 1073858416646707$

Lin, P., Fang, Z., Liu, J., and Lee, J. H. (2016). Optogenetic functional MRI. J. Vis. Exp. 110:53346. doi: $10.3791 / 53346$

Logothetis, N. K. (2008). What we can do and what we cannot do with fMRI. Nature 453, 869-878. doi: 10.1038/ nature 06976

Logothetis, N. K., Pauls, J., Augath, M., Trinath, T., and Oeltermann, A. (2001). Neurophysiological investigation of the basis of the fMRI signal. Nature 412, 150-157. doi: 10.1038/35084005

Machery, E. (2012). Dissociations in neuropsychology and cognitive neuroscience. Philos. Sci. 79, 490-518. doi: 10.1086/668002

Machery, E. (2014). In defense of reverse inference. Br. J. Philos. Sci. 65, 251-267. doi: 10.1093/bjps/axs044

National Research Council Federal Judicial Center Policy and Global Affairs Committee on Science T Law and Committee on the Development of the Third Edition of the Reference Manual on Scientific Evidence (2011). Reference Manual on Scientific Evidence, 3rd Edn. National Academies Press.

Nathan, M. J., and Del Pinal, G. (2017). The Future of Cognitive Neuroscience? Reverse Inference in Focus. Philos. Compass 12:e12427. doi: $10.1111 /$ phc3.12427

Nielsen, F. A., Hansen, L. K., and Balslev, D. (2004). Mining for associations between text and brain activation in a functional neuroimaging database. Neuroinformatics 2, 369-380. doi: 10.1385/NI:2:4:369 
Nitsche, M. A., Cohen, L. G., Wassermann, E. M., Priori, A., Lang, N., Antal, A., et al. (2008). Transcranial direct current stimulation: state of the art 2008. Brain Stimul. 1, 206-223. doi: 10.1016/j.brs.2008.06.004

Noppeney, U., Friston, K. J., and Price, C. J. (2004). Degenerate neuronal systems sustaining cognitive functions. J. Anat. 205, 433-442. doi: 10.1111/j.0021-8782.2004.00343.x

Pardo, M. S., and Patterson, D. (2011). Minds, brains, and norms. Neuroethics 4, 179-190. doi: 10.1007/s12152-010-9082-4

Poldrack, R. A. (2006). Can cognitive processes be inferred from neuroimaging data? Trends Cogn. Sci. 10, 59-63. doi: 10.1016/j.tics.2005.12.004

Poldrack, R. A. (2011). Inferring mental states from neuroimaging data: from reverse inference to large-scale decoding. Neuron 72, 692-697. doi: 10.1016/j.neuron.2011.11.001

Poldrack, R. A., and Yarkoni, T. (2016). From brain maps to cognitive ontologies: informatics and the search for mental structure. Annu. Rev. Psychol. 67, 587-612. doi: 10.1146/annurev-psych-122414-033729

Presidential Commission for the Study of Bioethical Issues (2015). Gray Matters: Topics at the Intersection of Neuroscience, Ethics, and Society, Vol. 2. Washington, DC: Bioethics.gov. Available online at: https://bioethicsarchive. georgetown.edu/pcsbi/sites/default/files/GrayMatter_V2_508.pdf (Accessed July 17, 2017).

Rubin, T. N., Koyejo, O., Gorgolewski, K. J., Jones, M. N., Poldrack, R. A., and Yarkoni, T. (2016). Decoding brain activity using a large-scale probabilistic functional-anatomical atlas of human cognition. bioRxiv. doi: 10.1101/059618

Ruff, C. C., Driver, J., and Bestmann, S. (2009). Combining, T. M. S., and fMRI: from 'virtual lesions' to functional-network accounts of cognition. Cortex 45, 1043-1049. doi: 10.1016/j.cortex.2008.10.012

Salerno, J. M., and Bottoms, B. L. (2009). Emotional evidence and jurors' judgments: the promise of neuroscience for informing psychology and law. Behav. Sci. Law 27, 273-296. doi: 10.1002/bsl.861

Seghier, M. L., Lee, H. L., Schofield, T., Ellis, C. L., and Price, C. J. (2008). Inter-subject variability in the use of two different neuronal networks for reading aloud familiar words. Neuroimage 42, 1226-1236. doi: 10.1016/j.neuroimage.2008.05.029

Shallice, T. (1988). From Neuropsychology to Mental Structure. Cambridge: Cambridge University Press.

Siebner, H. R., Bergmann, T. O., Bestmann, S., Massimini, M., JohansenBerg, H., Mochizuki, H., et al. (2009). Consensus paper: combining transcranial stimulation with neuroimaging. Brain Stimul. 2, 58-80. doi: $10.1016 /$ j.brs.2008.11.002
Sliwinska, M. W., Vitello, S., and Devlin, J. T. (2014). Transcranial magnetic stimulation for investigating causal brain-behavioral relationships and their time course. J. Vis. Exp. e51735. doi: 10.3791/ 51735

Sternberg, S. (2011). Modular processes in mind and brain. Cogn. Neuropsychol. 28, 156-208. doi: 10.1080/02643294.2011.557231

Steude, A., Witts, E. C., Miles, G. B., and Gather, M. C. (2016). Arrays of microscopic organic LEDs for high-resolution optogenetics. Sci. Adv. 2:e1600061. doi: 10.1126/sciadv.1600061

Teuber, H. L. (1955). Physiological psychology. Annu. Rev. Psychol. 6, 267-296. doi: 10.1146/annurev.ps.06.020155.001411

Tufail, Y., Matyushov, A., Baldwin, N., Tauchmann, M. L., Georges, J., Yoshihiro, A., et al. (2010). Transcranial pulsed ultrasound stimulates intact brain circuits. Neuron 66, 681-694. doi: 10.1016/j.neuron.2010. 05.008

Tufail, Y., Yoshihiro, A., Pati, S., Li, M. M., and Tyler, W. J. (2011). Ultrasonic neuromodulation by brain stimulation with transcranial ultrasound. Nat. Protoc. 6, 1453-1470. doi: 10.1038/nprot.2011.371

Wagner, T., Valero-Cabre, A., and Pascual-Leone, A. (2007). Noninvasive human brain stimulation. Annu. Rev. Biomed. Eng. 9, 527-565. doi: 10.1146/annurev.bioeng.9.061206.133100

Wang, S., Kugelman, T., Buch, A., Herman, M., Han, Y., Karakatsani, M. E., et al. (2017). Non-invasive, focused ultrasound-facilitated gene delivery for optogenetics. Sci. Rep. 7:39955. doi: 10.1038/srep39955

Woodward, J. (2005). Making Things Happen: A Theory of Causal Explanation. New York, NY: Oxford University Press.

Yarkoni, T., Poldrack, R. A., Nichols, T. E., Van Essen, D. C., and Wager, T. D. (2011). Large-scale automated synthesis of human functional neuroimaging data. Nat. Methods 8, 665-670. doi: 10.1038/nmeth.1635

Conflict of Interest Statement: The authors declare that the research was conducted in the absence of any commercial or financial relationships that could be construed as a potential conflict of interest.

Copyright (c) 2018 Jun and Yoo. This is an open-access article distributed under the terms of the Creative Commons Attribution License (CC BY). The use, distribution or reproduction in other forums is permitted, provided the original author(s) and the copyright owner are credited and that the original publication in this journal is cited, in accordance with accepted academic practice. No use, distribution or reproduction is permitted which does not comply with these terms. 